\title{
Design of Grating-Assisted Waveguide Couplers with Weighted Coupling
}

\author{
Kim A. Winick, Member, IEEE
}

\begin{abstract}
The design of grating-assisted, channel waveguide, codirectional couplers is demonstrated using the Gel'fand-Levitan-Marchenko inverse scattering method. Weighted coupling coefficients are computed from rational function expressions for the desired wavelength response. Approximate formulas are derived which relate the waveguide and grating geometries to the computed coupling coefficients. The technique is illustrated by designing a directional coupler having a third-order Butterworth filter characteristic and a $130 \AA$ full-width-half-maximum bandwidth.
\end{abstract}

\section{INTRODUCTION}

L IGHTWAVE communication networks have enor$L_{\text {mous information carrying capacities. Many users }}$ may share the network by using dense wavelength division multiplexing. These lightwave networks, along with other optoelectronic systems, require narrow-band wavelength-selective devices for successful operation. It has long been known that directional couplers, consisting of a pair of closely spaced, single-mode, parallel waveguides, may be used as filters [1]-[4]. Power is exchanged between the waveguides through the evanescent fields of the two guided modes. The transfer of power is described by the following relationship [5]:

$$
\frac{P_{2}(L)}{P_{1}(0)}=\frac{c_{12}^{2}}{\delta^{2}+c_{12} c_{21}} \sin ^{2}\left(\sqrt{\delta^{2}+c_{12} c_{21}} L\right) .
$$

In (1), $P_{1}(0)$ denotes the power injected into guide 1 at $z$ $=0$, and $P_{2}(L)$ equals the power transferred from guide 1 into guide 2 at a distance $z=L . c_{12}$ and $c_{21}$ are the coupling constants determined by the waveguide materials and geometry, and $\delta$ is the dephasing term

$$
2 \delta(\lambda)=\beta_{1}(\lambda)-\beta_{2}(\lambda)
$$

where $\beta_{1}(\lambda)$ and $\beta_{2}(\lambda)$ are the propagation constants of the combined waveguide structure, and $\lambda$ is wavelength. It is seen from (1) that maximum power transfer occurs when $\delta=0$ and $L\left(c_{12} c_{21}\right)^{1 / 2}=\pi / 2$. Using (1) and (2) the fractional bandwidth of the coupler may be expressed as

$$
\frac{\Delta \lambda}{\lambda_{0}}=\frac{0.8}{L}\left[\left(\frac{d N_{1}}{d \lambda}-\frac{d N_{2}}{d \lambda}\right)_{\lambda=\lambda_{0}}\right]^{-1}
$$

Manuscript received December 3, 1990; revised June 19, 1991. This work was supported in part by NSF grant ECS- 8909802

The author is with the Department of Electrical Engineering and Computer Science, University of Michigan, Ann Arbor, Ml 48109

IEEE Log Number 9102583. where $\Delta \lambda$ is the full-width-half-maximum filter bandwidth, $N_{1}$ and $N_{2}$ are the effective indexes of the two waveguide modes, $L\left(c_{12} c_{21}\right)^{1 / 2}=\pi / 2$, and $\lambda_{0}$ is the wavelength for which phase synchronism is achieved, that is $\delta=0$. For practical materials and coupling geometries, $d N_{1} / d \lambda-d N_{2} / d \lambda$ is typically quite small, therefore the selectivity of these filters is limited. In addition, the filters exhibit high sidelobe levels (i.e., $-9.3 \mathrm{~dB}$ ) as indicated in Fig. 1.

The selectivity of directional couplers may be increased significantly by phase matching the guides using a grating, as shown in Fig. 2. With grating-assisted coupling, (2) becomes

$$
2 \delta(\lambda)=\beta_{1}(\lambda) \pm \beta_{2}(\lambda)-\frac{2 \pi}{\Lambda}
$$

where $\Lambda$ is the grating period, and the \pm sign corresponds to the case of contradirectional and codirectional coupling, respectively. Similarly, (3) is modified to read

$$
\frac{\Delta \lambda}{\lambda_{0}}=\frac{0.8 \Lambda}{L}\left[1-\Lambda\left(\frac{d N_{1}}{d \lambda} \pm \frac{d N_{2}}{d \lambda}\right)_{\lambda=\lambda_{0}}\right]^{-1}
$$

where

$$
\Lambda=\frac{\lambda_{0}}{N_{1}\left(\lambda_{0}\right) \pm N_{2}\left(\lambda_{0}\right)}
$$

As indicated by (5) and (6), contradirectional coupling offers much greater wavelength selectivity than is achievable with codirectional operation. Contradirectional coupling, however, requires submicron period gratings, which may be difficult to fabricate. Recently, Alferness et al. [6] have demonstrated a codirectional, grating-assisted, coupler having a $65-\AA$ bandwidth. This device consisted of vertically stacked InGaAsP waveguides on InP, and phase matching was achieved using a relatively course 14.4- $\mu \mathrm{m}$ period grating. The device, however, exhibited high sidelobe levels.

It has been previously shown that filter characteristics may be controlled in corrugated waveguide filters (CWF) [7]-[12] and directional couplers by tapering the coupling coefficient [13]-[16]. In directional couplers, the tapering can be implemented by varying the spacing between the guides [13]-[15] or by varying grating parameters [16], such as length, depth, duty cycle, and/or period. Most of the work reported to date in this area has been directed 


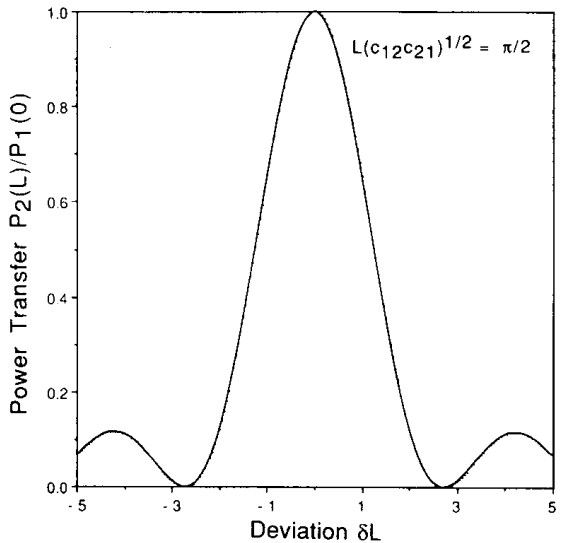

Fig. 1. Directional coupler response.

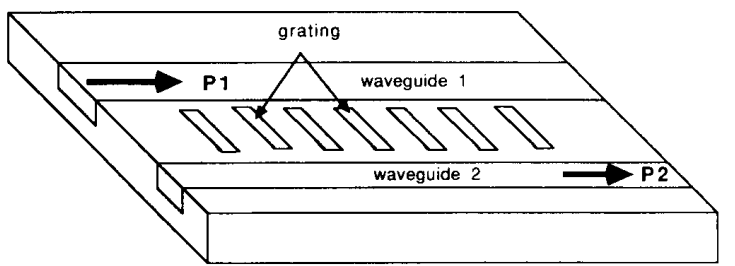

Fig. 2. Grating assisted directional coupler.

toward sidelobe suppression. Furthermore, only the strength of the coupling coefficient has been tapered (not the period), and the design techniques used have been ad hoc. Recently, Song and Shin [11] have applied the Gel'fand-Levitan-Marchenko inverse-scattering technique to the design of corrugated waveguide filters. Given a rational representation of the desired filter characteristic, their technique yields an exact analytic description of the required coupling coefficient. Song and Shin have applied this technique to design linear and Butterworth CWF's, while Winick and Roman [12] have used the method to design parabolic CWF's.

The fabrication of tapered corrugated waveguide filters suffers from practical difficulties. Since device operation is based on contradirectional mode coupling, submicron gratings are required. Codirectional, grating-assisted, directional couplers, on the other hand, need only coarse gratings. The corresponding increase in grating period should make it substantially easier to realize a specified amplitude and phase taper, thus achieving the desired coupling coefficient. Directional coupler filters also enjoy the advantage (compared to single guide CWF's) that the filtered wavelength components are spatially separated. Codirectional operation, however, does entail a sacrifice in wavelength selectivity as noted above, and may also result in significant coupling to radiation modes [17].

In this paper, codirectional, grating-assisted, directional couplers will be analyzed. The analysis will be restricted to planar waveguide geometries, consisting of a pair of parallel, asymmetric, channel waveguides, similar to those which may be fabricated in glass and $\mathrm{LiNbO}_{3}$ by ion or proton exchange. Coupling via both surface relief and bulk gratings imbedded between the guides will be considered, but radiation losses are not explicitly computed. Marcatili's method will be used to determine the modes of the combined structure. The coupling coefficient will be expressed as an integral involving the mode profiles and the grating parameters. An approximate closed-form expression for the coupling coefficient will also be derived in the limit of negligible guide-to-guide interaction. Finally, these results will be combined with the Gel'fand-Levitan-Marchenko inverse scattering technique to design a directional coupler, which has a thirdorder Butterworth filter characteristic.

\section{Field Distribution and Propagation Constants for Parallel Channel Waveguides}

Directional couplers have been constructed using a variety of geometries. Two of the more common configurations are a pair of parallel channel waveguides imbedded into a planar substrate (see Fig. 2), and a pair of vertically stacked planar waveguides [6]. The vertically stacked configuration is particularly well-suited for monolithically integrated optoelectronic devices, fabricated using epitaxially grown semiconductor materials. Confinement, however, is restricted only to a plane, propagation losses are high, and the growth process is complex. Parallel channel waveguides, on the other hand, can be easily fabricated in $\mathrm{LiNbO}_{3}$, glass, and semiconductor materials. Furthermore, in glass and $\mathrm{LiNbO}_{3}$ substrates, low-loss channel guides are obtained using standard photolithography and thermal ion exchange or proton exchange techniques. Single-step fabrication of both the channel waveguides and the phase matching grating is also a possibility when using these materials.

In this section the field distribution and the propagation constants will be computed (approximately) for an imbedded pair of parallel, equal depth, rectangular, channel waveguides. The computation is based on a direct application of Marcatili's method, which can be used when the guided modes are not near cutoff.

Consider the directional coupler shown in Fig. 3. This device is divided into the following nine regions:

$$
\begin{array}{ll}
\text { region } 1:-d \leq x \leq 0 ; & 0 \leq y \leq w_{1} \\
\text { region 2: } x \leq-d ; & 0 \leq y \leq w_{1} \\
\text { region 3: } 0 \leq x ; & 0 \leq y \leq w_{1} \\
\text { region 4: }-d \leq x \leq 0 ; & w_{1} \leq y \\
\text { region 5: }-d \leq x \leq 0 ; & -s \leq y \leq 0 \\
\text { region } 1^{\prime}:-d \leq x \leq 0 ; & -\left(s+w_{2}\right) \leq y \leq-s \\
\text { region 2': } x \leq-d ; & -\left(s+w_{2}\right) \leq y \leq-s \\
\text { region } 3^{\prime}: 0 \leq x ; & -\left(s+w_{2}\right) \leq y \leq-s \\
\text { region } 4^{\prime}:-d \leq x \leq 0 ; & y \leq-\left(s+w_{2}\right) .
\end{array}
$$




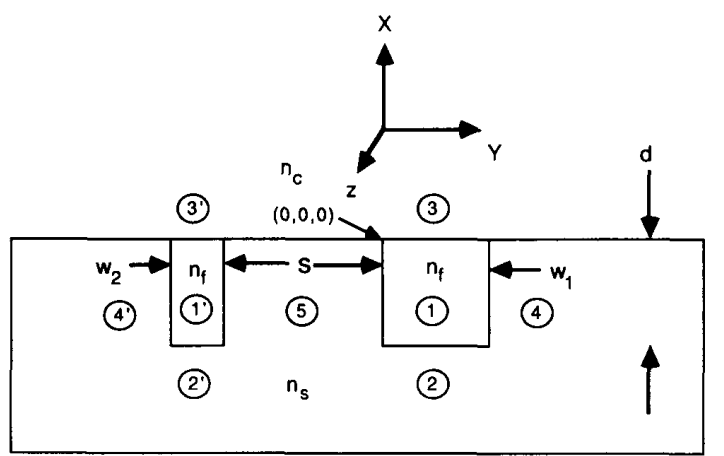

Fig. 3. Directional coupler geometry (end view, grating not shown).

It is assumed that the guided modes are not near cutoff, and thus the electric and magnetic fields will be small outside of these nine regions and may be neglected. Using Maxwell's equations, the transverse electric and magnetic field components can be expressed in terms of the longitudinal field components as follows:

$$
\begin{aligned}
& E_{x}=\left(-i / K_{m}^{2}\right)\left[\beta \frac{\partial E_{z}}{\partial x}+\omega \mu_{0} \frac{\partial H_{z}}{\partial y}\right] \\
& E_{y}=\left(-i / K_{m}^{2}\right)\left[\beta \frac{\partial E_{z}}{\partial y}-\omega \mu_{0} \frac{\partial H_{z}}{\partial x}\right] \\
& H_{x}=\left(-i / K_{m}^{2}\right)\left[\beta \frac{\partial H_{z}}{\partial x}-\omega n_{m}^{2} \epsilon_{0} \frac{\partial E_{z}}{\partial y}\right] \\
& H_{y}=\left(-i / K_{m}^{2}\right)\left[\beta \frac{\partial H_{z}}{\partial y}+\omega n_{m}^{2} \epsilon_{0} \frac{\partial E_{z}}{\partial x}\right]
\end{aligned}
$$

where $i=(-1)^{1 / 2}, k=2 \pi / \lambda, n_{m}$ is the refractive index in the $m$ th region, $\beta$ is the propagation constant of a guided mode of the combined two waveguide structure, and

$$
K_{m}^{2}=\left(k^{2} n_{m}^{2}-\beta^{2}\right) .
$$

The longitudinal components $E_{z}$ and $H_{z}$ must satisfy the scalar wave equation

$$
\frac{\partial \psi^{2}}{\partial x^{2}}+\frac{\partial \psi^{2}}{\partial y^{2}}+K_{m}^{2} \psi=0
$$

We restrict our analysis to propagating modes, whose electric fields are aligned primarily along the $y$-direction (i.e., quasi-TE modes). It is easy to verify that the field components given in Appendix A satisfy (7) and (8), and yield such a mode. For guiding to occur, the propagation constants must lie in the range

$$
k n_{s} \leq \beta \leq k n_{f} \text {. }
$$

Assuming that $n_{s} \approx n_{f}$, it follows from (A1)-(A10) in Appendix A that

$$
\begin{aligned}
& E_{x} \ll E_{z} \ll E_{y} \\
& H_{z} \ll H_{x} \text { and } H_{y}=0 .
\end{aligned}
$$

Thus, the dominant field components are $E_{y}, E_{z}, H_{x}$, and $H_{z}$.

The dispersion relations of the guided modes can now be determined by matching the tangential $E$ and $H$ fields at the boundaries separating the regions. Observe that (1) $E_{y}$ is already approximately matched at the $1: 3,1: 2$, $1^{\prime}: 3^{\prime}$, and $1^{\prime}: 2^{\prime}$ boundaries (since $\kappa_{y} \ll k n_{f}, \kappa_{y} \ll k n_{s}$, $\kappa_{y} \ll k n_{c}$ ), (2) $E_{z}$ is exactly matched at the $1: 4,1: 5$, $1^{\prime}: 5$, and $1^{\prime}: 4^{\prime}$ boundaries, (3) $E_{z}$ is approximately matched at the $1: 2$ and $1^{\prime}: 2^{\prime}$ boundaries (since $n_{f} \approx n_{s}$ ), and (4) $E_{z}$ may be neglected at the remaining $1: 3$ and $1^{\prime}: 3^{\prime}$ boundaries (since $E_{z} \ll E_{y}$ ). After some tedious calculations, it can be shown that matching the tangential $H_{z}$ component at the $1: 2,1: 3,1^{\prime}: 2^{\prime}$, and $1^{\prime}: 3^{\prime}$ boundaries yields (12) below

$V_{x} \sqrt{1-b_{x}}=p \pi+\tan ^{-1} \sqrt{\frac{b_{x}}{1-b_{x}}}+\tan ^{-1} \sqrt{\frac{b_{x}+a}{1-b_{x}}}$

where

$$
\begin{aligned}
V_{x} & =k d \sqrt{n_{f}^{2}-n_{s}^{2}} \\
b_{x} & =\frac{N_{x}^{2}-n_{s}^{2}}{n_{f}^{2}-n_{s}^{2}} \\
a & =\frac{n_{s}^{2}-n_{c}^{2}}{n_{f}^{2}-n_{s}^{2}} \\
\beta_{x} & =k N_{x} \\
\kappa_{x}^{2} & =k^{2} n_{f}^{2}-\beta_{x}^{2}
\end{aligned}
$$

and $p$ is a nonnegative integer. Similarly, matching the tangential $H_{x}$ and $H_{z}$ at the remaining $1: 4,1: 5 ; 1^{\prime}: 5$, and $1^{\prime}: 4^{\prime}$ boundaries yields

$$
\begin{aligned}
{\left[\frac{n_{s}^{2}}{n_{f}^{2}} \sqrt{\frac{1-b_{y}}{b_{y}}}+\frac{n_{f}^{2}}{n_{s}^{2}} \sqrt{\frac{b_{y}}{1-b_{y}}}\right] \exp \left(-2 \frac{s V_{y}}{w_{1}} \sqrt{b_{y}}\right) } \\
\cdot \tan \left(V_{y} \sqrt{1-b_{y}}\right) \tan \left(\frac{w_{2}}{w_{1}} V_{y} \sqrt{1-b_{y}}\right) \\
=4\left[1-\frac{1}{2}\left(\frac{n_{s}^{2}}{n_{f}^{2}} \sqrt{\frac{1-b_{y}}{b_{y}}}-\frac{n_{f}^{2}}{n_{s}^{2}} \sqrt{\frac{b_{y}}{1-b_{y}}}\right)\right. \\
\left.\cdot \tan \left(V_{y} \sqrt{1-b_{y}}\right)\right] \\
\times\left[1-\frac{1}{2}\left(\frac{n_{s}^{2}}{n_{f}^{2}} \sqrt{\frac{1-b_{y}}{b_{y}}}-\frac{n_{f}^{2}}{n_{s}^{2}} \sqrt{\frac{b_{y}}{1-b_{y}}}\right)\right. \\
\left.\cdot \tan \left(\frac{w_{2}}{w_{1}} V_{y} \sqrt{1-b_{y}}\right)\right]
\end{aligned}
$$


where

$$
\begin{aligned}
V_{y} & =k w_{1} \sqrt{n_{f}^{2}-n_{s}^{2}} \\
b_{y} & =\frac{N_{y}^{2}-n_{s}^{2}}{n_{f}^{2}-n_{s}^{2}} \\
\beta_{y} & =k N_{y} \\
\kappa_{y}^{2} & =k^{2} n_{f}^{2}-\beta_{y}^{2} .
\end{aligned}
$$

Note that (18) will always have at least two solutions. The propagation constant of the entire two guide structure may be expressed in terms of $b_{x}$ and $b_{y}$ by combining (14), (16), (17), (20)-(22), and (Alg). The resulting expression is

$$
\beta^{2}=\left(n_{f}^{2}-n_{s}^{2}\right)\left(b_{x}+b_{y}-1\right)+n_{s}^{2} .
$$

Observe that (12)-(17) are identical to those describing TE mode propagation in a three layer asymmetrical slab waveguide having refractive indexes $n_{c}, n_{f}, n_{s}$, and thicknesses $\infty, d, \infty$. Similarly, (18)-(22) describe TM mode propagation in a pair of parallel slab waveguides, consisting of five layers having refractive indexes $n_{s}, n_{f}, n_{s}$, $n_{f}, n_{s}$, and thicknesses $\infty, w_{1}, s, w_{2}, \infty$.

When the separation $s$ between the waveguides is made sufficiently large, then guides no longer show significant interaction. With $s=\infty$, the left-hand side of (18) vanishes, and the right-hand side reduces to the following pair of independent equations:

$$
\begin{gathered}
V_{y} \sqrt{1-b_{y}}=q \pi+2 \tan ^{-1} \frac{n_{f}^{2}}{n_{s}^{2}} \sqrt{\frac{b_{y}}{1-b_{y}}} \\
\frac{w_{2}}{w_{1}} V_{y} \sqrt{1-b_{y}}=r \pi+2 \tan ^{-1} \frac{n_{f}^{2}}{n_{s}^{2}} \sqrt{\frac{b_{y}}{1-b_{y}}}
\end{gathered}
$$

where $q$ and $r$ are nonnegative integers. Fig. 4 is a plot of the effective indexes $\beta / k$, of the first two waveguide modes as a function of the waveguide separation $s$. Fig. 5 is a plot of the corresponding mode profiles, $H_{x}(y)$. The field strengths have been normalized so that the two modes shown have equal power. For comparison purposes, the mode profiles are also plotted in Fig. 5 assuming that the two individual guides do not interact. It follows from Figs. 4 and 5 that the individual waveguides (for the example shown) are essentially noninteracting when separated by more than approximately $1 \mu \mathrm{m}$. The electric field profile $E_{y}(y)$ can be determined from $H_{x}(y)$ using the field expressions given in Appendix A.

Each of the separate waveguides should support only a single mode. This places upper and lower limits on $V_{x}$ and $V_{y}$. Since cutoff "along the $x$ direction" occurs when $b_{x}$ $=0$, it follows from (12) that $V_{x}$ must satisfy

$$
\tan ^{-1} \sqrt{a} \leq V_{x} \text {. }
$$

Observe from (23) that $b_{y}$ must be greater than $1-b_{x}$ for guiding to occur (i.e., for $\beta>k n_{s}$ ). Combining this fact

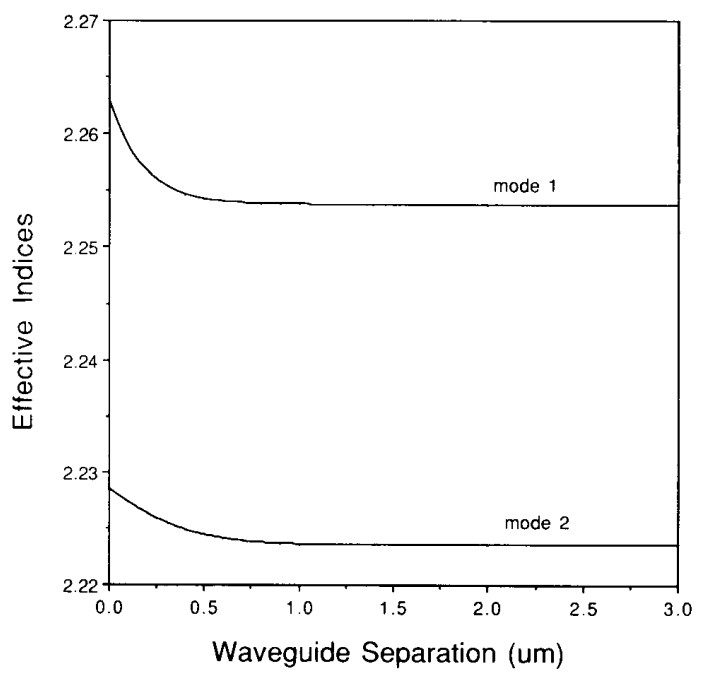

Fig. 4. Effective indexes of the guided modes $\left(n_{f}=2.3, n_{s}=2.2, n_{c}=\right.$ $\left.1.0, d=w_{1}=1.5 \mu \mathrm{m}, w_{2}=0.7 \mu \mathrm{m}, s=2.0 \mu \mathrm{m}\right)$.

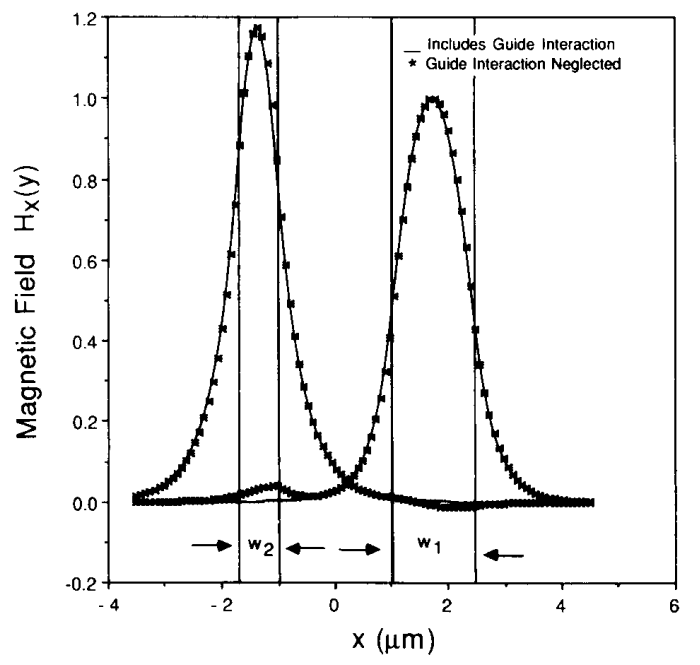

Fig. 5. Magnetic field mode profiles $\left(n_{f}=2.3, n_{s}=2.2, n_{c}=1.0, d=\right.$ $\left.w_{1}=1.5 \mu \mathrm{m}, w_{2}=0.7 \mu \mathrm{m}, s=2.0 \mu \mathrm{m}\right)$.

(and $\left.V_{y}=\left(w_{i} / d\right) V_{x}\right)$ with (24) yields the following bound for the aspect ratios, $w_{i} / d$, of the waveguides:

$$
\begin{gathered}
\frac{\pi}{V_{x} \sqrt{b_{x}}}+\frac{2 \tan ^{-1}\left(\frac{n_{f}^{2}}{n_{s}^{2}} \sqrt{\frac{1-b_{x}}{b_{x}}}\right)}{V_{x} \sqrt{b_{x}}} \\
\geq \frac{w_{i}}{d} \geq \frac{2 \tan ^{-1}\left(\frac{n_{f}^{2}}{n_{s}^{2}} \sqrt{\frac{1-b_{x}}{b_{x}}}\right)}{V_{x} \sqrt{b_{x}}} \quad i=1 \text { or } 2
\end{gathered}
$$




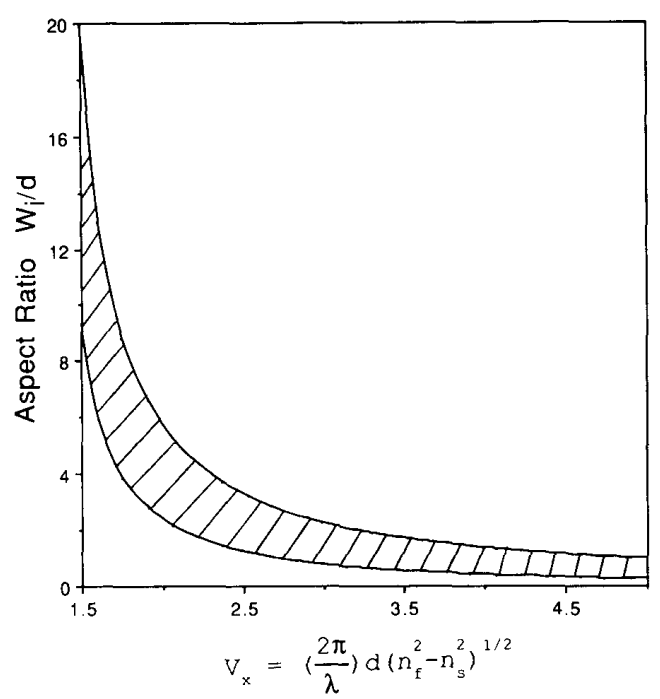

Fig. 6. Single-mode propagation region $\left(n_{f}=2.3, n_{s}=2.2, n_{c}=1.0\right)$.

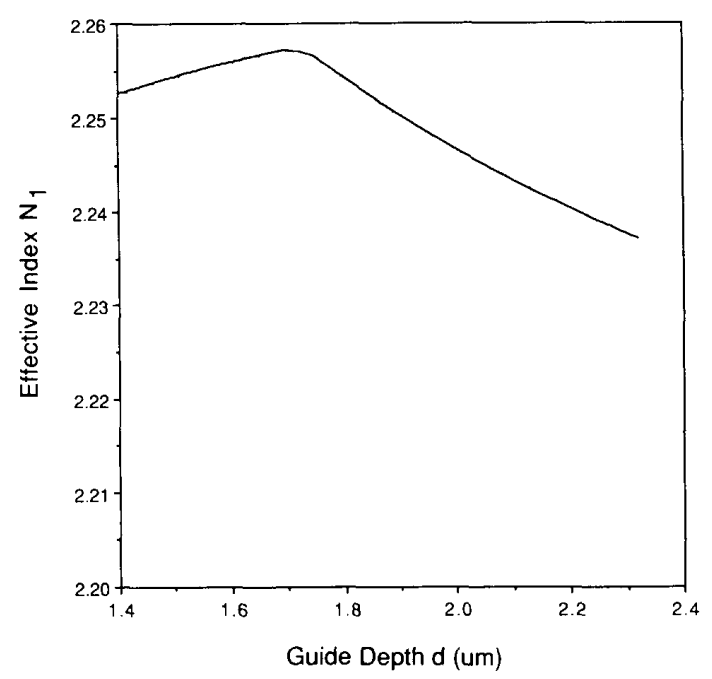

Fig. 7. Maximum effective index for a single-mode guide $\left(n_{f}=2.3, n_{\mathrm{s}}=\right.$ $2.2, n_{c}=1.0$ ).

where $b_{x}$ is given by (12) with $p=0$. If for $p=1,(12)$ admits a second solution, denoted $b_{x}^{\prime}$, then for singlemode operation the aspect ratio must also satisfy (27b) below

$$
\frac{w_{i}}{d} \leq \frac{2 \tan ^{-1}\left(\frac{n_{f}^{2}}{n_{s}^{2}} \sqrt{\frac{1-b_{x}^{\prime}}{b_{x}^{\prime}}}\right)}{V_{x} \sqrt{b_{x}^{\prime}}} \quad i=1 \text { or } 2 .
$$

Using (23), (26), and (27), the allowable $V_{x}-w_{i} / d$ region for single-mode operation is computed and plotted in Fig. 6.
It follows from (5) and (6) that the grating-assisted codirectional coupler will have the largest wavelength selectivity when the effective indexes, $N_{1}$ and $N_{2}$, have the largest difference. This difference is at its maximum when one of the guided modes is as far from cutoff as possible, while the other lies very close. When the waveguides do not interact significantly then max $\left(N_{1}\right)-N_{2}$ may be computed with the aid of Fig. 6. Fig. 7 is a plot of $\max \left(N_{1}\right)$ versus $d$ for single mode operation. Note that $N_{1}-N_{2}$ must always be less than $N_{1}-n_{s}$, which in turn is less than $n_{f}-n_{s}$. Thus, material considerations will significantly impact the achievable wavelength selectivity.

\section{Field Normalization}

The expressions for $E_{y}$ and $H_{x}$ can be simplified when the guide-to-guide separation, $s$, is large. The fields of the two guided modes $(m=1,2)$ can then be written as

$$
\begin{aligned}
& E_{y, m}(x, y) \approx E_{y, m}(x) f_{m}(y) \\
& H_{x, m}(x, y) \approx H_{x, m}(x) f_{m}(y)=\frac{-\left(\frac{\epsilon_{0}}{\mu_{0}}\right)^{1 / 2}}{k} \beta_{x} E_{y, m}(x) f_{m}(y)
\end{aligned}
$$

where

$$
E_{y, m}(x)= \begin{cases}C_{m} \cos \kappa_{x} \phi \exp \left(-\gamma_{3} x\right), & 0 \leq x \\ C_{m} \cos \kappa_{x}(x+\phi), & 0 \geq x \geq-d \\ C_{m} \cos \kappa_{x}(\phi-d) \exp \left[\gamma_{2}(x+d)\right], & x \leq-d\end{cases}
$$

$$
f_{1}(y)=\left\{\begin{array}{lc}
\frac{n_{s}^{2}}{n_{f}^{2}} \frac{\kappa_{y, 1}}{\gamma_{4,1}} \sin \left(\kappa_{y, 1} w_{1} / 2\right) & \exp \left[-\gamma_{4,1}\left(y-w_{1}\right)\right], \\
y \geq w_{1} \\
\cos \kappa_{y, 1}\left(y-w_{1} / 2\right), & 0 \leq y \leq w_{1} \\
\frac{n_{s}^{2}}{n_{f}^{2}} \frac{\kappa_{y, 1}}{\gamma_{4,1}} \sin \left(\kappa_{y, 1} w_{1} / 2\right) & \exp \left(\gamma_{4,1} y\right), \\
y \leq 0
\end{array}\right.
$$

$$
f_{2}(y)=\left\{\begin{array}{c}
\frac{n_{s}^{2}}{n_{f}^{2}} \frac{\kappa_{y, 2}}{\gamma_{4,2}} \sin \left(\kappa_{y, 2} w_{2} / 2\right) \exp \left[-\gamma_{4,2}(y+s)\right], \\
y \geq-s \\
\cos \kappa_{y, 2}\left(y+s+w_{2} / 2\right), \\
-\left(s+w_{2}\right) \leq y \leq-s \\
\frac{n_{s}^{2} \frac{\kappa_{y, 2}}{n_{f}^{2}} \sin \left(\kappa_{y, 2} w_{2} / 2\right) \exp \left[\gamma_{4,2}\left(y+s+w_{2}\right)\right],}{y \leq-\left(s+w_{2}\right)}
\end{array}\right.
$$




$$
C_{m}=\frac{i A k^{2} n_{f}^{2}}{\kappa_{y, m} \beta_{x}} .
$$

The second subscript in $\gamma_{4, m}$, and $\kappa_{y, m}$ denotes one of the two guided modes, that is, either $m=1$ or $m=2$. Equations (28)-(32) were derived using the field expressions given in Appendix $\mathrm{A},(\mathrm{A} 10)$, and the fact that $\beta \approx \beta_{x}$ when $n_{f} \approx n_{s}$.

If $\boldsymbol{P}_{m}$ equals the power in the $m$ th guided mode, then

$$
\begin{aligned}
2 P_{m} & =-\int_{-\infty}^{\infty} \int_{-\infty}^{\infty} E_{y, m}(x, y) H_{x, m}(x, y) d x d y \\
& =-\int_{-\infty}^{\infty} E_{y, m}(x) H_{x, m}(x) d x \int_{-\infty}^{\infty} f_{m}^{2}(y) d y .
\end{aligned}
$$

With $P_{m}$ set to $1 / 2$, it is shown in Appendix B that

$$
C_{m}^{2} \approx 4\left(\frac{\mu_{0}}{\epsilon_{0}}\right)^{1 / 2} \frac{1}{N_{x} d_{\mathrm{eff}} W_{\mathrm{eff}, m}}
$$

where

$$
\begin{aligned}
d_{\mathrm{eff}} & =d+\frac{1}{\gamma_{2}}+\frac{1}{\gamma_{3}} \\
w_{\mathrm{eff}, m} & =w_{m}+\frac{n_{s}^{4}}{n_{f}^{4}} \frac{\kappa_{y, m}^{2}}{\gamma_{4, m}^{2}} \frac{2}{\gamma_{4, m}} .
\end{aligned}
$$

Note that $N_{x}, \gamma_{2}$, and $\gamma_{3}$ do not depend on $w_{1}$ or $w_{2}$. From (30) and (A11), it is also shown that

$$
\begin{aligned}
\int_{-d}^{0} & E_{y, 1}(x) E_{y \cdot 2}(x) d x \\
\quad= & \frac{C_{1} C_{2}}{2}\left[d+\frac{\left(N_{x}^{2}-n_{c}^{2}\right)^{1 / 2}}{k\left(n_{f}^{2}-n_{c}^{2}\right)}+\frac{\left(N_{x}^{2}-n_{s}^{2}\right)^{1 / 2}}{k\left(n_{f}^{2}-n_{s}^{2}\right)}\right] .
\end{aligned}
$$

IV. Mode Coupling

It is well known that the interaction between the two orthogonal guided modes of a directional coupler can be described by the following pair of first-order differential equations [5]:

$$
\begin{aligned}
& \frac{d B_{1}(z)}{d z}=-i c_{12}(z) \exp [i 2 \delta(\lambda) z] B_{2}(z) \\
& \frac{d B_{2}(z)}{d z}=-i c_{12}^{*}(z) \exp [-i 2 \delta(\lambda) z] B_{1}(z)
\end{aligned}
$$

where $B_{1}(z)$ and $B_{2}(z)$ are the complex electric field amplitudes of the two guided modes, $c_{12}(z)$ is the coupling coefficient, and $\delta(\lambda)$ is defined by (4)

$$
2 \delta(\lambda)=\beta_{1}(\lambda)-\beta_{2}(\lambda)-\frac{2 \pi}{\Lambda} .
$$

In the grating region, the refractive index $n_{g}(x, y, z)$ of the directional coupler may be expressed as

$n_{g}^{2}(x, y, z)=n_{s}^{2}+\Delta n^{2}(x, y, z) \operatorname{sgn}\left[\cos \left(\frac{2 \pi}{\Lambda} z+\Omega(z)\right)\right]$ where

$$
\operatorname{sgn}(u)= \begin{cases}0, & u \leq 0 \\ 1, & u>0 .\end{cases}
$$

The coupling coefficient is then given by [5]

$$
\begin{aligned}
c_{12}(z)= & \frac{\exp (-i \Omega(z))}{\lambda\left(\mu_{0} / \epsilon_{0}\right)^{1 / 2}} \int_{-\infty}^{\infty} \int_{-\infty}^{\infty} \Delta n^{2}(x, y, z) \\
& \cdot\left[E_{y, 1}(x, y) E_{y 2}(x, y)+E_{x 1}(x, y) E_{x 2}(x, y)\right. \\
& \left.+\frac{n_{s}^{2}}{n_{g}^{2}(x, y, z)} E_{z 1}(x, y) E_{z 2}(x, y)\right] d x d y
\end{aligned}
$$

where $\left(E_{x, m}, E_{y, m}, E_{z, m}\right)$ is the normalized (i.e., $P_{m}=1 / 2$ in (33)) electric field amplitude of the $m$ th guided mode, and $\Delta n^{2}(x, y, z)$ is the grating-induced refractive index perturbation. Note that the term $\Omega(z)$ in (42) is used to describe nonperiodic gratings.

The $x$ and $z$ field components in (42) may be neglected since $E_{x} \ll E_{z} \ll E_{y}$ (see (10)). Thus

$$
\begin{aligned}
c_{12}(z)= & \frac{\exp (-i \Omega(z))}{\lambda\left(\mu_{0} / \epsilon_{0}\right)^{1 / 2}} \int_{-\infty}^{\infty} \int_{-\infty}^{\infty} \Delta n^{2}(x, y, z) \\
& \cdot\left[E_{y \cdot 1}(x, y) E_{y 2}(x, y)\right] d x d y .
\end{aligned}
$$

If the grating is centered between the two waveguides, has depth $d$, width $t(z)$, and a uniform index change $\Delta n^{2}=$ $n_{f}^{2}-n_{s}^{2}$, then (43) becomes

$$
\begin{aligned}
c_{12}(z) \approx & \frac{\exp [-i \Omega(z)]}{\lambda\left(\mu_{0} / \epsilon_{0}\right)^{1 / 2}}\left(n_{f}^{2}-n_{s}^{2}\right) \int_{-d}^{0} \int_{-(s+t(z)) / 2}^{-(s-t(z)) / 2} \\
& \cdot E_{y, 1}(x, y) E_{y, 2}(x, y) d x d y .
\end{aligned}
$$

The coupling coefficient may be evaluated numerically using the field expressions given in Appendix A. When the guides do not have significant interaction, then the simplified field expressions given in Section III may be used instead, and the following result is obtained:

$$
\begin{aligned}
c_{12}(z) \approx & t(z) D \frac{n_{s}^{4}}{n_{f}^{4}} \frac{2}{\lambda N_{x}} \sqrt{\left(n_{f}^{2}-N_{y, 1}^{2}\right)\left(n_{f}^{2}-N_{y, 2}^{2}\right)} \\
& \cdot \frac{1}{\sqrt{w_{\text {eff. } 1} w_{\text {eff }, 2}}} \exp \left[-\left(\gamma_{4,1}+\gamma_{4,2}\right) s / 2\right] \\
& \times \frac{\sinh \left[\left(\gamma_{4,1}-\gamma_{4,2}\right) t(z) / 2\right]}{\left(\gamma_{4,1}-\gamma_{4,2}\right) t(z) / 2}
\end{aligned}
$$

where

$$
D=\left[d+\frac{\sqrt{N_{x}^{2}-n_{c}^{2}}}{k\left(n_{f}^{2}-n_{c}^{2}\right)}+\frac{\sqrt{N_{x}^{2}-n_{s}^{2}}}{k\left(n_{f}^{2}-n_{s}^{2}\right)}\right] d_{\mathrm{eff}}^{-1} .
$$

Note that the above coupled mode analysis assumes that the guided modes of the separate channel waveguides are approximately orthogonal [18]. If the grating is of the sur- 
face relief, rather than bulk type, then $c_{12}(z)$ given above must be multiplied by the following factor:

$$
\frac{2 h}{D d_{\text {eff }}} \frac{\left(n_{s}^{2}-n_{c}^{2}\right)\left(n_{f}^{2}-N_{x}^{2}\right)}{\left(n_{f}^{2}-n_{s}^{2}\right)\left(n_{f}^{2}-n_{c}^{2}\right)}
$$

where $h$ is the depth of the surface corrugation.

\section{Gel'Fand-Levitan-Marchenko Technique}

The Gel'fand-Levitan-Marchenko (GLM) inverse scattering technique was developed for quantum mechanics. In principle, it provides an exact method for finding the potential function in Schrodinger's wave equation from scattering data. The potential is obtained as the solution of an integral equation, and for one-dimensional problems, this integral equation reduces to a set of linear simultaneous equations. Kay [19], and later Pechenick and Cohen [20], showed that these equations may be easily solved numerically, provided the scattering data is expressed as a rational function of wavelength. The GLM technique can be extended to inverse scattering problems, where the scattering system is modeled as a pair of coupled-mode equations, such as those given in (39). In these cases, the objective is to find $c_{12}(z)$, given measurements of $B_{2}(L) / B_{1}(L)$ versus $\delta$. As in the one-dimensional problem, the GLM integral equations can be easily solved numerically, provided the scattering data, $B_{2}(L) / B_{1}(L)$, is expressed as a rational function of $\delta$ [11].

The GLM procedure is implemented as follows. First define the reflection coefficient, $r(\delta)$, as $B_{2}(L) / B_{1}(L)$ evaluated at $\delta$. Second, let $u=-i \delta$ and express $r(u)$ as a rational function $G(u)=P(u) / Q(u) . Q(u)$ is a polynomial of $\operatorname{order} N$ with all of its roots lying in the left half plane, and $P(u)$ is a polynomial of order $N-1$ or less. Third, find the $N$ roots of $Q(u)$ in the complex plane and denote them by $\rho_{1}, \rho_{2}, \cdots, \rho_{N}$. Fourth, form the polynomial $F(u)=Q(u) Q^{*}\left(-u^{*}\right)+P(u) P^{*}\left(-u^{*}\right)$, and find the $2 N$ roots of $F(u)$ in the complex plane. Denote these roots by $\omega_{1}, \omega_{2}, \cdots, \omega_{N},-\omega_{1}^{*},-\omega_{2}^{*}, \cdots,-\omega_{N}^{*}$. Fifth, solve the following system of $2 N$ simultaneous linear equations:

$$
\begin{gathered}
\sum_{m=1}^{N}\left\{\frac{1}{G\left(\omega_{m}\right)} \frac{\exp \left(-\omega_{m} z\right)}{\rho_{n}-\omega_{m}}\left[\begin{array}{l}
g_{1, m}(z) \\
g_{2, m}(z)
\end{array}\right]\right. \\
\left.-\frac{\exp \left(\omega_{m}^{*} z\right)}{\rho_{n}+\omega_{m}^{*}}\left[\begin{array}{r}
g_{2, m}^{*}(z) \\
-g_{1, m}^{*}(z)
\end{array}\right]\right\}=\left[\begin{array}{l}
1 \\
0
\end{array}\right] \\
(n=1,2, \cdots, N)
\end{gathered}
$$

for $g_{1, m}(z)$, and $g_{2, m}(z)$, where $z$ denotes distance measured along the length of the grating from $z=0$ to $z=$ $L$. Finally, compute the coupling coefficient using (49) below.

$$
\begin{aligned}
c_{12}(z)= & 2 \sum_{n=1}^{N} g_{1, n}(z) \exp \left(\omega_{n} z\right) \\
& -G\left(-\omega_{n}^{*}\right) g_{2, n}^{*}(z) \exp \left(-\omega_{n}^{*} z\right) .
\end{aligned}
$$

Observe from (39) that the reflection and coupling coefficients scale according to the following rule:

If $\delta_{c}$ is an arbitrary constant and if $c_{12}(z)$ corresponds

$$
\text { to } r(\delta) \text {, then } \delta_{c} c_{12}(z) \text { corresponds } r\left(\delta / \delta_{c}\right) \text {. }
$$

Also note that

$$
r^{*}(\delta)=-r(-\delta) .
$$

As an example, consider the directional coupler with parameters specified in Table I. We wish to determine the grating profile needed to realize a third-order Butterworth filter response, with a 130 - $\AA$ full-width-half-maximum bandwidth. Thus

$$
\left|B_{2}(L)\right|^{2}=\frac{b^{2}}{1+\left(\delta / \delta_{c}\right)^{6}}
$$

where $\delta_{c}$ is the filter half-power point, and $b^{2}$ is the fraction of input power transfered to guide 2 at phase synchronism (i.e., $\delta=0$ ). Without loss of generality, it has been assumed that $B_{1}(0)=1$. In view of $(50)$, we may also assume that $\delta_{c}=1$ and consider scaling factors later. If the device is lossless, then power must be conserved and thus

$$
\left|B_{1}(L)\right|^{2}+\left|B_{2}(L)\right|^{2}=1 .
$$

Combining (51), (52), and (53) yields

$|G(u)|^{2}=G^{*}(u) G(u)=-G(-u) G(u)=\frac{b^{2}}{\left(1-b^{2}\right)-u^{6}}$.

Thus, $P(u), Q(u)$, and $F(u)$ are given by

$$
\begin{aligned}
& P(u)=i b \\
& Q(u)=\prod_{k=2}^{4}\left(-u+\left(1-b^{2}\right)^{1 / 6} \exp \left[i \frac{\pi}{3} k\right]\right) \\
& F(u)=1-u^{6} .
\end{aligned}
$$

If $b=0.9$, it then follows that

$$
\begin{aligned}
& \rho_{1}=0.7582 \exp \left(i \frac{2 \pi}{3}\right), \quad \rho_{2}=-0.7582, \\
& \rho_{3}=0.7582 \exp \left(i \frac{4 \pi}{3}\right) \\
& \omega_{1}=1, \quad \omega_{2}=\exp \left(i \frac{\pi}{3}\right), \quad \omega_{3}=\exp \left(-i \frac{\pi}{3}\right) .
\end{aligned}
$$

Fig. 8 is a plot of $c_{12}(z)$ obtained from (48) and (49). The corresponding plot of $\left|B_{2}(L)\right|^{2}$, computed from (39) with $c_{12}(z)$ truncated to the interval $0 \leq z \leq 6$, is shown in Fig. 9. For comparison purposes, the desired filter response $0.81 /\left(1+\delta^{2}\right)$ is also shown. Note the excellent agreement.

It follows from (4) and (6) that

$$
\begin{aligned}
2 \delta(\lambda) \approx & \frac{-2 \pi\left(N_{1}-N_{2}\right)}{\lambda_{0}^{2}}\left[1-\Lambda\left(\frac{d N_{1}}{d \lambda}-\frac{d N_{2}}{d \lambda}\right)_{\lambda=\lambda_{0}}\right] \\
& \cdot\left(\lambda-\lambda_{0}\right) .
\end{aligned}
$$




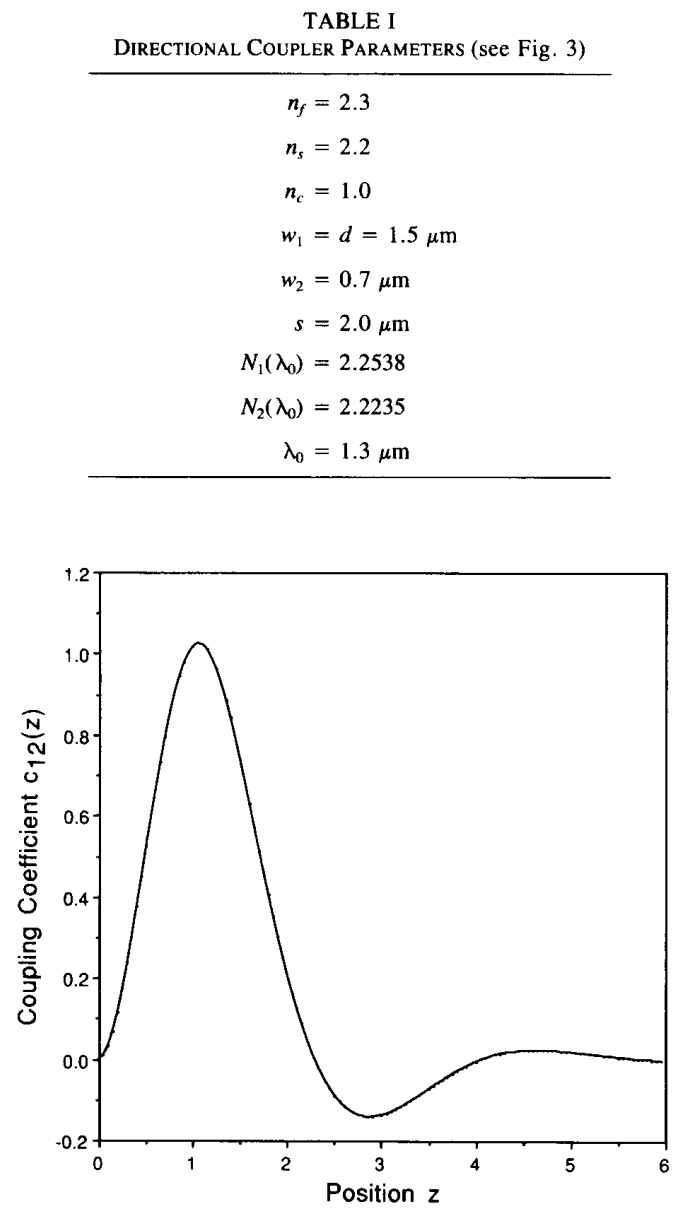

Fig. 8. Coupling coefficient for 3rd-order butterworth filter (cut-off normalized to 1 , maximum magnitude $=0.81$ ).

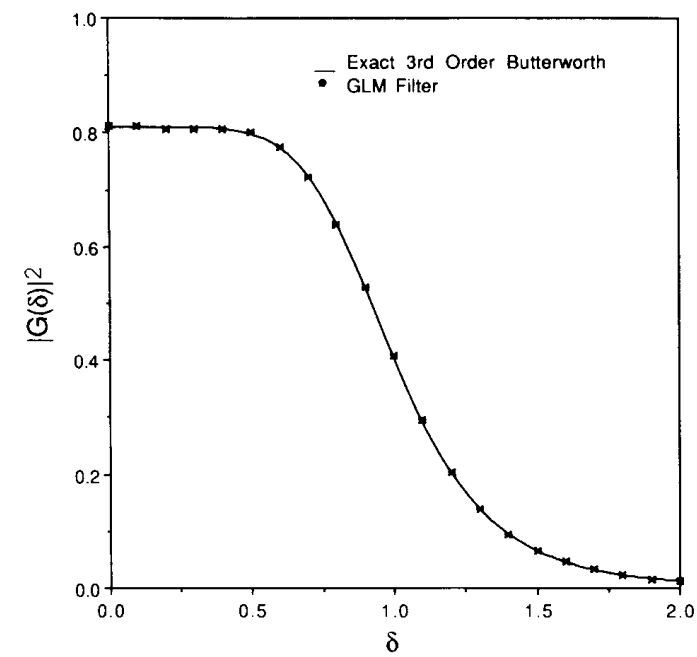

Fig. 9. 3rd-order butterworth filter design using GLM technique.

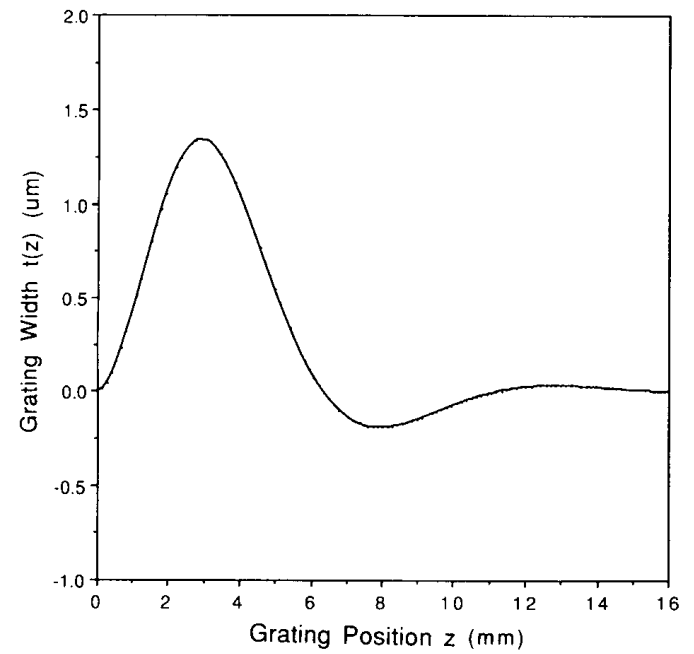

Fig. 10. Tapered grating widths required for a 3rd-order butterworth filter (bandwidth $130 \AA$, maximum magnitude $=0.81$ ).

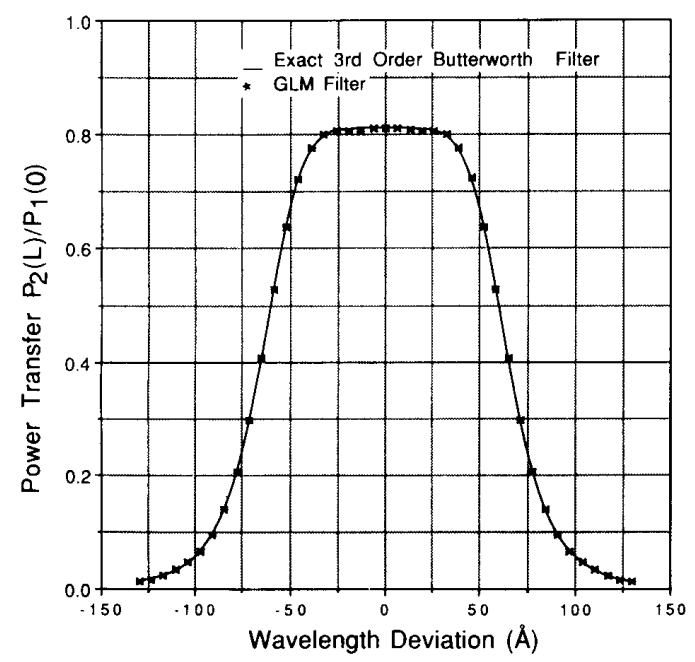

Fig. 11. 3rd-order butterworth filter designed using GLM technique.

For glass and $\mathrm{LiNbO}_{3}$, the dispersion term on (58) is small and thus

$$
2 \delta(\lambda) \approx-\frac{2 \pi}{\lambda_{0}^{2}}\left(N_{1}-N_{2}\right)\left(\lambda-\lambda_{0}\right)
$$

Using the coupler parameters given in Table I, and assuming a FWHM of $130 \AA$, it follows from (59) that $\delta_{c}$ $=0.36 \mathrm{~mm}^{-1}$. Fig. 10 is a plot of the required grating width, $t(z)$, computed from the Fig. 8 and (45). A negative value of $t(z)$ is used to indicate a $180^{\circ}$ phase shift in the grating. Physically, this phase shift is realized by displacing the grating by one-half cycle at the position $z$, where $t$ goes to zero. Fig. 11 gives the filter response corresponding to Fig. 10 . 
Finally, observe that it may be easier to produce a desired coupling coefficient by modulating the duty cycle of the grating, rather than by changing its width, $t$. This is true because the grating period is quite large compared to its width.

\section{Conclusions}

In this paper, the design of grating-assisted, codirectional couplers is demonstrated using the Gel'fand-Levitan-Marchenko inverse scattering technique. The couplers considered are parallel, step-index, channel waveguides, separated by either bulk or surface relief gratings. Weighted coupling coefficients are computed, given a specification of the coupler's wavelength response. The waveguide and grating geometries are related to these coupling coefficients using approximate, closedform expressions, which are derived. The technique is illustrated by designing a proton-exchanged, $\mathrm{LiNbO}_{3}$, directional coupler, which has a third-order Butterworth filter characteristic and a 130 - $\AA$ full-width-half-maximum bandwidth.

In grating assisted codirectional couplers, the grating periods are rather large. This fact should make it possible to fabricate some of these devices using standard photolithography, combined with ion diffusion or proton exchange techniques. Large grating periods, however, do limit the achievable wavelength selectivity and may lead to significant radiation losses. The design technique discussed in this paper may be easily extended to contradirectional coupling, which greatly enhances the selectivity and eliminates the radiation problem. Device fabrication, however, becomes difficult, because tapered submicron gratings are required.

\section{APPENDIX A}

Region 1:

$$
\begin{aligned}
E_{z}= & A \cos \kappa_{x}(x+\phi) \cos \kappa_{y}(y+\eta) \quad \text { (Ala) } \\
H_{z}= & A\left(\frac{\epsilon_{0}}{\mu_{0}}\right)^{1 / 2} n_{f}^{2}\left(\frac{\kappa_{x}}{\kappa_{y}}\right)\left(\frac{k}{\beta}\right) \\
& \cdot \sin \kappa_{x}(x+\phi) \sin \kappa_{y}(y+\eta) \quad \text { (Alb) } \\
E_{x}= & -i A\left(\frac{\kappa_{x}}{\beta}\right) \sin \kappa_{x}(x+\phi) \cos \kappa_{y}(y+\eta) \quad \text { (Alc) } \\
E_{y}= & i A\left(\frac{1}{\kappa_{y} \beta}\right)\left(k^{2} n_{f}^{2}-\kappa_{y}^{2}\right) \cos \kappa_{x}(x+\phi) \sin \kappa_{y}(y+\eta) \\
H_{x}= & -i A\left(\frac{\epsilon_{0}}{\mu_{0}}\right)^{1 / 2} n_{f}^{2}\left(\frac{k}{\kappa_{y}}\right) \cos \kappa_{x}(x+\phi) \sin \kappa_{y}(y+\eta)
\end{aligned}
$$

$$
H_{y}=0
$$

where

$$
k^{2} n_{f}^{2}-\beta^{2}=\kappa_{x}^{2}+\kappa_{y}^{2} .
$$

Region 2:

$$
\begin{aligned}
& E_{z}=A\left(\frac{n_{f}^{2}}{n_{s}^{2}}\right) \cos \kappa_{x}(\phi-d) \cos \kappa_{y}(y+\eta) \\
& \cdot \exp \left[\gamma_{2}(x+d)\right] \\
& H_{z}=-A\left(\frac{\epsilon_{0}}{\mu_{0}}\right)^{1 / 2} n_{f}^{2}\left(\frac{\gamma_{2}}{\kappa_{y}}\right)\left(\frac{k}{\beta}\right) \cos \kappa_{x}(\phi-d) \\
& \cdot \sin \kappa_{y}(y+\eta) \exp \left[\gamma_{2}(x+d)\right] \\
& E_{x}=i A\left(\frac{\gamma_{2}}{\beta}\right)\left(\frac{n_{f}^{2}}{n_{s}^{2}}\right) \cos \kappa_{x}(\phi-d) \cos \kappa_{y}(y+\eta) \\
& \cdot \exp \left[\gamma_{2}(x+d)\right] \\
& E_{y}=i A\left(\frac{1}{\kappa_{y} \beta}\right) \frac{n_{f}^{2}}{n_{s}^{2}}\left(k^{2} n_{s}^{2}-\kappa_{y}^{2}\right) \cos \kappa_{x}(\phi-d) \\
& \cdot \sin \kappa_{y}(y+\eta) \exp \left[\gamma_{2}(x+d)\right] \\
& H_{x}=-i A\left(\frac{\epsilon_{0}}{\mu_{0}}\right)^{1 / 2} n_{f}^{2}\left(\frac{k}{\kappa_{y}}\right) \cos \kappa_{x}(\phi-d) \\
& \cdot \sin \kappa_{y}(y+\eta) \exp \left[\gamma_{2}(x+d)\right] \\
& H_{y}=0
\end{aligned}
$$

where

$$
k^{2} n_{s}^{2}-\beta^{2}=\kappa_{y}^{2}-\kappa_{2}^{2} .
$$

Region 3:

$$
E_{z}=A\left(\frac{n_{f}^{2}}{n_{c}^{2}}\right) \cos \kappa_{x} \phi \cos \kappa_{y}(y+\eta) \exp \left(-\gamma_{3} x\right)
$$

$$
\begin{aligned}
H_{z}= & A\left(\frac{\epsilon_{0}}{\mu_{0}}\right)^{1 / 2} n_{f}^{2}\left(\frac{\gamma_{3}}{\kappa_{y}}\right)\left(\frac{k}{\beta}\right) \\
& \cdot \cos \kappa_{x} \phi \sin \kappa_{y}(y+\eta) \exp \left(-\gamma_{3} x\right)
\end{aligned}
$$

$$
E_{x}=-i A\left(\frac{\gamma_{3}}{\beta}\right)\left(\frac{n_{f}^{2}}{n_{c}^{2}}\right) \cos \kappa_{x} \phi \cos \kappa_{y}(y+\eta)
$$$$
\text { - } \exp \left(-\gamma_{3} x\right)
$$$$
E_{y}=i A\left(\frac{1}{\kappa_{y} \beta}\right) \frac{n_{f}^{2}}{n_{c}^{2}}\left(k^{2} n_{c}^{2}-\kappa_{y}^{2}\right)
$$$$
\text { - } \cos \kappa_{x} \phi \sin \kappa_{y}(y+\eta) \exp \left(-\gamma_{3} x\right)
$$

$$
H_{x}=-i A\left(\frac{\epsilon_{0}}{\mu_{0}}\right)^{1 / 2} n_{f}^{2}\left(\frac{k}{\kappa_{y}}\right)
$$$$
\text { - } \cos \kappa_{x} \phi \sin \kappa_{y}(y+\eta) \exp \left(-\gamma_{3} x\right)
$$

$H_{y}=0$ 
where

$$
k^{2} n_{c}^{2}-\beta^{2}=\kappa_{y}^{2}-\gamma_{3}^{2} .
$$

Region 4:

$E_{z}=A \cos \kappa_{y}\left(w_{1}+\eta\right) \cos \kappa_{x}(x+\phi) \exp \left[-\gamma_{4}\left(y-w_{1}\right)\right]$

(A4a)

$$
H_{z}=-A\left(\frac{\epsilon_{0}}{\mu_{0}}\right)^{1 / 2} n_{s}^{2}\left(\frac{\kappa_{x}}{\gamma_{4}}\right)\left(\frac{k}{\beta}\right) \cos \kappa_{y}\left(w_{1}+\eta\right)
$$$$
\cdot \sin \kappa_{x}(x+\phi) \exp \left[-\gamma_{4}\left(y-w_{1}\right)\right]
$$$$
E_{x}=-i A\left(\frac{\kappa_{x}}{\beta}\right) \cos \kappa_{y}\left(w_{1}+\eta\right) \sin \kappa_{x}(x+\phi)
$$$$
\cdot \exp \left[-\gamma_{4}\left(y-w_{1}\right)\right]
$$

(A4b)

$$
E_{y}=-i A\left(\frac{1}{\gamma_{4} \beta}\right)\left(k^{2} n_{s}^{2}+\gamma_{4}^{2}\right) \cos \kappa_{y}\left(w_{1}+\eta\right)
$$$$
\cdot \cos \kappa_{x}(x+\phi) \exp \left[-\gamma_{4}\left(y-w_{1}\right)\right]
$$

$$
H_{x}=i A\left(\frac{\epsilon_{0}}{\mu_{0}}\right)^{1 / 2} n_{s}^{2}\left(\frac{k}{\gamma_{4}}\right) \cos \kappa_{y}\left(w_{1}+\eta\right)
$$$$
\cdot \cos \kappa_{x}(x+\phi) \exp \left[-\gamma_{4}\left(y-w_{1}\right)\right]
$$

$H_{y}=0$

where

\section{Region 5:}

$$
\begin{aligned}
E_{z}= & A \cos \kappa_{y} \eta \cos \kappa_{x}(x+\phi) \frac{\cosh \gamma_{4}(y+\alpha)}{\cosh \gamma_{4} \alpha} \\
H_{z}= & A\left(\frac{\epsilon_{0}}{\mu_{0}}\right)^{1 / 2} n_{s}^{2}\left(\frac{\kappa_{x}}{\gamma_{4}}\right)\left(\frac{k}{\beta}\right) \\
& \cdot \cos \kappa_{y} \eta \sin \kappa_{x}(x+\phi) \frac{\sinh \gamma_{4}(y+\alpha)}{\cosh \gamma_{4} \alpha} \\
E_{x}= & -i A\left(\frac{\kappa_{x}}{\beta}\right) \cos \kappa_{y} \eta \sin \kappa_{x}(x+\phi) \frac{\cosh \gamma_{4}(y+\alpha)}{\cosh \gamma_{4} \alpha}
\end{aligned}
$$$$
E_{y}=i A\left(\frac{1}{\gamma_{4} \beta}\right)\left(k^{2} n_{s}^{2}+\gamma_{4}^{2}\right)
$$$$
\cdot \cos \kappa_{y} \eta \cos \kappa_{x}(x+\phi) \frac{\sinh \gamma_{4}(y+\alpha)}{\cosh \gamma_{4} \alpha}
$$$$
H_{x}=-i A\left(\frac{\epsilon_{\theta}}{\mu_{0}}\right)^{1 / 2} n_{s}^{2}\left(\frac{k}{\gamma_{4}}\right)
$$$$
\cdot \cos \kappa_{y} \eta \cos \kappa_{x}(x+\phi) \frac{\sinh \gamma_{4}(y+\alpha)}{\cosh \gamma_{4} \alpha}
$$

$H_{y^{\prime}}=0$.

(A5c)

(A5d)

(A5e)

\section{Region 1':}

$$
E_{z}=A^{\prime} \cos \kappa_{x}(x+\phi) \cos \kappa_{y}\left(y+\eta^{\prime}\right)
$$

$$
H_{z}=A^{\prime}\left(\frac{\epsilon_{0}}{\mu_{0}}\right)^{1 / 2} n_{f}^{2}\left(\frac{\kappa_{x}}{\kappa_{y}}\right)\left(\frac{k}{\beta}\right)
$$

$$
\cdot \sin \kappa_{x}(x+\phi) \sin \kappa_{y}\left(y+\eta^{\prime}\right)
$$

$$
E_{x}=-i A^{\prime}\left(\frac{\kappa_{x}}{\beta}\right) \sin \kappa_{x}(x+\phi) \cos \kappa_{y}\left(y+\eta^{\prime}\right)
$$

$$
E_{y}=i A^{\prime}\left(\frac{1}{\kappa_{y} \beta}\right)\left(k^{2} n_{f}^{2}-\kappa_{y}^{2}\right)
$$

$$
\cdot \cos \kappa_{x}(x+\phi) \sin \kappa_{y}\left(y+\eta^{\prime}\right)
$$

$$
H_{x}=-i A^{\prime}\left(\frac{\epsilon_{0}}{\mu_{0}}\right)^{1 / 2} n_{f}^{2}\left(\frac{k}{\kappa_{y}}\right)
$$

$$
\cdot \cos \kappa_{x}(x+\phi) \sin \kappa_{y}\left(y+\eta^{\prime}\right)
$$

$$
H_{y}=0
$$

where

$$
A^{\prime}=\frac{\cos \left(\kappa_{y} \eta\right) \cos \gamma_{4}(s-\alpha)}{\cosh \left(\gamma_{4} \alpha\right) \cos \kappa_{y}\left(\eta^{\prime}-s\right)}
$$

(A6g)

Region 2':

$E_{z}=A^{\prime}\left(\frac{n_{f}^{2}}{n_{y}^{2}}\right) \cos \kappa_{x}(\phi-d) \cos \kappa_{y}\left(y+\eta^{\prime}\right)$

$\cdot \exp \left[\gamma_{2}(x+d)\right]$

$$
H_{z}=-A^{\prime}\left(\frac{\epsilon_{0}}{\mu_{0}}\right)^{1 / 2} n_{f}^{2}\left(\frac{\gamma_{2}}{\kappa_{y}}\right)\left(\frac{k}{\beta}\right) \cos \kappa_{x}(\phi-d)
$$$$
\cdot \sin \kappa_{y}\left(y+\eta^{\prime}\right) \exp \left[\gamma_{2}(x+d)\right]
$$

$$
E_{x}=i A^{\prime}\left(\frac{\gamma_{2}}{\beta}\right)\left(\frac{n_{f}^{2}}{n_{s}^{2}}\right) \cos \kappa_{x}(\phi-d) \cos \kappa_{y}\left(y+\eta^{\prime}\right)
$$$$
\cdot \exp \left[\gamma_{2}(x+d)\right]
$$

$$
\begin{aligned}
E_{y}= & i A^{\prime}\left(\frac{1}{\kappa_{y} \beta}\right) \frac{n_{f}^{2}}{n_{s}^{2}}\left(k^{2} n_{s}^{2}-\kappa_{y}^{2}\right) \cos \kappa_{x}(\phi-d) \\
& \cdot \sin \kappa_{y}\left(y+\eta^{\prime}\right) \exp \left[\gamma_{2}(x+d)\right] \\
H_{x}= & -i A^{\prime}\left(\frac{\epsilon_{0}}{\mu_{0}}\right)^{1 / 2} n_{f}^{2}\left(\frac{k}{\kappa_{y}}\right) \cos \kappa_{x}(\phi-d) \\
& \cdot \sin \kappa_{y}\left(y+\eta^{\prime}\right) \exp \left[\gamma_{2}(x+d)\right] \\
H_{y}= & 0 .
\end{aligned}
$$

Region 3':

$E_{z}=A^{\prime}\left(\frac{n_{f}^{2}}{n_{c}^{2}}\right) \cos \kappa_{x} \phi \cos \kappa_{y}\left(y+\eta^{\prime}\right) \exp \left(-\gamma_{3} x\right)$

$(\mathrm{A} 8 \mathrm{a})$ 


$$
\begin{aligned}
H_{z}= & A^{\prime}\left(\frac{\epsilon_{0}}{\mu_{0}}\right)^{1 / 2} n_{f}^{2}\left(\frac{\gamma_{3}}{\kappa_{y}}\right)\left(\frac{k}{\beta}\right) \\
& \cdot \cos \kappa_{x} \phi \sin \kappa_{y}\left(y+\eta^{\prime}\right) \exp \left(-\gamma_{3} x\right) \\
E_{x}= & -i A\left(\frac{\gamma_{3}}{\beta}\right)\left(\frac{n_{f}^{2}}{n_{c}^{2}}\right) \cos \kappa_{x} \phi \cos \kappa_{y}\left(y+\eta^{\prime}\right) \\
& \cdot \exp \left(-\gamma_{3} x\right) \\
E_{y}= & i A^{\prime}\left(\frac{1}{\kappa_{y} \beta}\right) \frac{n_{f}^{2}}{n_{c}^{2}}\left(k^{2} n_{c}^{2}-\kappa_{y}^{2}\right) \\
& \cdot \cos \kappa_{x} \phi \sin \kappa_{y}\left(y+\eta^{\prime}\right) \exp \left(-\gamma_{3} x\right) \\
H_{x}= & -i A^{\prime}\left(\frac{\epsilon_{0}}{\mu_{0}}\right)^{1 / 2} n_{f}^{2}\left(\frac{k}{\kappa_{y}}\right) \cos \kappa_{x} \phi \sin \kappa_{y}\left(y+\eta^{\prime}\right) \\
& \cdot \exp \left(-\gamma_{3} x\right) \\
H_{y}= & 0 .
\end{aligned}
$$

Region 4':

$$
\begin{aligned}
E_{z}= & A^{\prime} \cos \kappa_{y}\left(\eta^{\prime}-s-w_{2}\right) \cos \kappa_{x}(x+\phi) \\
& \cdot \exp \left[\gamma_{4}\left(y+s+w_{2}\right)\right] \\
H_{z}= & A^{\prime}\left(\frac{\epsilon_{0}}{\mu_{0}}\right)^{1 / 2} n_{s}^{2}\left(\frac{\kappa_{x}}{\gamma_{4}}\right)\left(\frac{k}{\beta}\right) \cos \kappa_{y} \eta^{\prime} \sin \kappa_{x}(x+\phi) \\
& \cdot \exp \left[\gamma_{4}\left(y+s+w_{2}\right)\right] \\
E_{x}= & -i A^{\prime}\left(\frac{\kappa_{x}}{\beta}\right) \cos \kappa_{y} \eta^{\prime} \sin \kappa_{x}(x+\phi) \\
& \cdot \exp \left[\gamma_{4}\left(y+s+w_{2}\right)\right] \\
E_{y}= & i A^{\prime}\left(\frac{1}{\gamma_{4} \beta}\right)\left(k^{2} n_{s}^{2}+\gamma_{4}^{2}\right) \cos \kappa_{y} \eta^{\prime} \cos \kappa_{x}(x+\phi) \\
& \cdot \exp \left[\gamma_{4}\left(y+s+w_{2}\right)\right] \\
H_{x}= & -i A^{\prime}\left(\frac{\epsilon_{0}}{\mu_{0}}\right)^{1 / 2} n_{s}^{2}\left(\frac{k}{\gamma_{4}}\right) \cos \kappa_{y} \eta^{\prime} \cos \kappa_{x}(x+\phi) \\
& \cdot \exp \left[\gamma_{4}\left(y+s+w_{2}\right)\right] \\
H_{y}= & 0
\end{aligned}
$$

The propagation constant, $\beta$, of a guided mode must satisfy the inequality $n_{s} \leq \beta \leq n_{f}$. Thus, it follows from (A1g), (A2g), and (A4g) that

$$
\begin{aligned}
& \kappa_{y} \ll \beta \\
& \kappa_{x} \ll \beta \\
& \kappa_{y} \ll k n_{f} \\
& \kappa_{x} \ll k n_{f} \\
& \gamma_{4} \ll k n_{f} \\
& \gamma_{2} \ll k n_{f}
\end{aligned}
$$

when $n_{s} \approx n_{f}$.

In (A1)-(A9), the parameters $\kappa_{x}, \kappa_{y}, \phi, \beta, \eta, \eta^{\prime}$, and $\alpha$ ( $A$ is an arbitrary constant) are determined by matching the tangential $E$ and $H$ fields at the boundaries of the nine regions. In particular, matching the tangential $H_{z}$ component at the $1: 2$ and $1: 3$ boundaries yields the following value for $\phi$ :

$$
\phi=\frac{1}{\kappa_{x}} \tan ^{-1} \frac{\gamma_{3}}{\kappa_{x}} .
$$

Similarly, matching the tangential $H_{x}$ at the $1: 4,1: 5$, and $1^{\prime}: 4^{\prime}$, yields $\eta, \eta^{\prime}$ and $\alpha$ as indicated below

$$
\begin{aligned}
\tan \kappa_{y}\left(w_{1}+\eta\right) & =-\frac{n_{s}^{2}}{n_{f}^{2}} \frac{\kappa_{y}}{\gamma_{4}} \\
\sin \kappa_{y}\left(s+w_{2}\right)-\cos \kappa_{y}\left(s+w_{2}\right) \tan \kappa_{y} \eta^{\prime} & =\frac{\kappa_{y}}{\gamma_{4}} \frac{n_{s}^{2}}{n_{f}^{2}} \\
\tan h \gamma_{4} \alpha & =\frac{\gamma_{4}}{\kappa_{y}} \tan \kappa_{y} \eta .
\end{aligned}
$$

Expressions for the remaining parameters $\kappa_{x}, \kappa_{y}$, and $\beta$ are given in Section II of this paper.

Below several useful identities are given. First, from (A1g), (A2g), (A3g), and (A4g) it follows that:

$$
\begin{aligned}
& k^{2}\left(n_{f}^{2}-n_{s}^{2}\right)=\kappa_{x}^{2}+\gamma_{2}^{2} \\
& k^{2}\left(n_{f}^{2}-n_{s}^{2}\right)=\kappa_{y}^{2}+\gamma_{4}^{2} \\
& k^{2}\left(n_{f}^{2}-n_{c}^{2}\right)=\kappa_{x}^{2}+\gamma_{3}^{2} .
\end{aligned}
$$

Second, using (16), (17), (21), and (22) one finds

$$
\begin{aligned}
& k^{2}\left(n_{f}^{2}-N_{y}^{2}\right)=\kappa_{y}^{2} \\
& k^{2}\left(n_{f}^{2}-N_{x}^{2}\right)=\kappa_{x}^{2}
\end{aligned}
$$

and finally, combining (A15) and (A16) yields

$$
\begin{aligned}
& k^{2}\left(N_{y}^{2}-n_{s}^{2}\right)=\gamma_{4}^{2} \\
& k^{2}\left(N_{x}^{2}-n_{s}^{2}\right)=\gamma_{2}^{2} \\
& k^{2}\left(N_{x}^{2}-n_{c}^{2}\right)=\gamma_{3}^{2} .
\end{aligned}
$$

\section{APPENDIX B}

Observe that $E_{y}(x)$ and $H_{x}(x)$, as given in (28)-(30), are identical to the TE mode field expressions for a three layer, asymmetrical, slab waveguide [21]. Therefore,

$$
-\int_{-\infty}^{\infty} E_{y, m}(x) H_{x, m}(x) d x=\frac{N_{x}}{2}\left(\frac{\epsilon_{0}}{\mu_{0}}\right)^{1 / 2} C_{m}^{2} d_{\mathrm{eff}}
$$

where

$$
d_{\mathrm{eff}}=d+\frac{1}{\gamma_{2}}+\frac{1}{\gamma_{3}}
$$

Direct integration of $f_{m}^{2}(y)$ yields 


$$
\begin{aligned}
\int_{-\infty}^{\infty} f_{m}^{2}(y) d y & \\
= & \frac{w_{m}}{2}+\frac{n_{s}^{4}}{n_{f}^{4}} \frac{\kappa_{y, m}^{2}}{\gamma_{4, m}^{2}}\left(\frac{\sin \left(\kappa_{y, m} w_{m} / 2\right) \cos \left(\kappa_{y, m} w_{m} / 2\right)}{\kappa_{y, m}}\right. \\
& \left.+\frac{\cos ^{2}\left(\kappa_{y, m} w_{m} / 2\right)}{\gamma_{4, m}}\right) .
\end{aligned}
$$

It follows from (A12) that

$$
\tan \left(\kappa_{y, m} w_{m} / 2\right)=\frac{n_{f}^{2}}{n_{s}^{2}}\left(\frac{\gamma_{4, m}}{\kappa_{y, m}}\right) .
$$

Thus,

$$
\sin \left(\kappa_{y, m} w_{m} / 2\right)=\frac{n_{f}^{2} \gamma_{4, m}}{\sqrt{n_{f}^{4} \gamma_{4, m}^{2}+n_{s}^{4} \kappa_{y, m}^{2}}} \approx \frac{\gamma_{4, m}}{\sqrt{\gamma_{4, m}^{2}+\kappa_{y, m}^{2}}}
$$

$$
\cos \left(\kappa_{y, m} w_{m} / 2\right)=\frac{n_{s}^{2} \kappa_{4, m}}{\sqrt{n_{f}^{4} \gamma_{4, m}^{2}+n_{s}^{4} \kappa_{y, m}^{2}}} \approx \frac{\kappa_{4, m}}{\sqrt{\gamma_{4, m}^{2}+\kappa_{y, m}^{2}}}
$$

where the approximations given in (B5) and (B6) follow from $n_{s} \approx n_{f}$. Combining (B3), (B5), and (B6) yields

$$
\int_{-\infty}^{\infty} f_{m}^{2}(y) d y \approx \frac{1}{2}\left(w_{m}+\frac{n_{s}^{4}}{n_{f}^{4}} \frac{\kappa_{y, m}^{2}}{\gamma_{4, m}^{2}} \frac{2}{\gamma_{4, m}}\right)
$$

Equations (35)-(37) in Section III now immediately follow from (B1), (B2), and (B7).

Direct integration of $E_{y}(x)$ given by $(30)$ yields

$$
\begin{aligned}
\int_{-d}^{0} E_{y, 1}(x) E_{y, 2}(x) d x \\
=\frac{C_{1} C_{2} d}{2}+\frac{C_{1} C_{2}}{2 \kappa_{x}}\left[\sin \kappa_{x} \phi \cos \kappa_{x} \phi\right. \\
\left.\quad+\sin \left(\kappa_{x}(d-\phi)\right) \cos \left(\kappa_{x}(d-\phi)\right)\right] .
\end{aligned}
$$

It follows from (A11) that

$$
\sin \kappa_{x} \phi \cos \kappa_{x} \phi=\frac{\kappa_{x} \gamma_{3}}{\gamma_{3}^{2}+\kappa_{x}^{2}}=\frac{\kappa_{x}\left(N_{x}^{2}-n_{c}^{2}\right)^{1 / 2}}{k\left(n_{f}^{2}-n_{c}^{2}\right)} .
$$

Observe that (12) and (13) can be written as

$$
\kappa_{x} d=\tan ^{-1} \frac{\gamma_{3}}{\kappa_{x}}+\tan ^{-1} \frac{\gamma_{2}}{\kappa_{x}}+p \pi .
$$

Combining (A11) with (B10) yields

$$
\begin{aligned}
& \sin \left(\kappa_{x}(d-\phi)\right) \cos \left(\kappa_{x}(d-\phi)\right) \\
& =\frac{\kappa_{x} \gamma_{2}}{\gamma_{2}^{2}+\kappa_{x}^{2}}=\frac{\kappa_{x}\left(N_{x}^{2}-n_{s}^{2}\right)^{1 / 2}}{k\left(n_{f}^{2}-n_{s}^{2}\right)} .
\end{aligned}
$$

Equation (38) of Section II now follows directly from (B8), (B9), and (B11).

\section{REFERENCES}

[1] E. A. Marcatili, Bell Syst. Tech. J., vol. 48, pp. 2071-2102, 1969.

[2] S. E. Miller, Bell Syst. Tech. J., vol. 48, pp. 2059-2069, 1969.

[3] D. Marcuse, Theory of Dielectric Optical Waveguides. San Diego, CA: Academic, 1974, ch. 1.

[4] H. F. Taylor, "Frequency-selective coupling in parallel dielectric waveguides," Opt. Commun., vol. 8, pp. 421-425, Aug. 1973.

[5] D. L. Lee, Electromagnetic Principles of Integrated Optics. New York: Wiley, 1986, ch. 8.

[6] R. C. Alferness et al., "Grating-assisted InGaAsP/InP vertical codirectional coupler filter," Appl. Phys. Lett., vol. 55, pp. 2011-2013, Nov. 1989.

[7] K. O. Hill, "Aperiodic distributed-parameter waveguides for integrated optics," Appl. Opt., vol, 13, pp. 1853-1856, Aug. 1974.

[8] H. Kogelnik, "Filter response of nonuniform almost-periodic structures," Bell Syst. Tech. J., vol. 55, pp. 109-126, Jan. 1976.

[9] J. B. Shellan, C. S. Hong, and A. Yariv, "Theory of chirped gratings in broadband filters," Opt. Commun., vol. 23, pp. 398-400, Dec. 1977.

[10] M. Matsuhara and K. O. Hill, "Optical waveguide band-rejection filters: Design,"' Appl. Opt., vol. 13, pp. 2886-2888, Dec. 1974.

[11] G. -H. Song and S. -Y. Shin, "Design of corrugated waveguide filters by the Gel'fand-Levitan-Marchenko inverse scattering method," $J$. Opt. Soc. Amer., vol. 2. pp. 1905-1915. Nov. 1985

[12] K. A. Winick and J. E. Roman, "Design of corrugated waveguide filters by Fourier transform techniques," IEEE J. Quantum Electron., vol. 26, pp. 1918-1929, Nov. 1990.

[13] T. Findakly and C. - L. Chen. "Optical directional couplers with variable spacing," Appl. Opt., vol. 17, pp. 769-773, Mar. 1978.

[14] R. C. Alferness and P. S. Cross, "Filter characteristics of codirectionally coupled waveguides with weighted coupling," IEEE $J$. Quantum Electron., vol. 14, pp. 843-847, Nov. 1978.

[15] R. C. Alferness, "Optical directional couplers with weighted coupling." Appl. Phys. Lett., vol. 35, pp. 260-262, Aug. 1979.

[16] P. Yeh and H. F. Taylor, "Contradirectional frequency-selective couplers for guided-wave optics," Appl. Opt., vol. 19, pp. 2848 2855, Aug. 1980

[17] D. Marcuse, "Radiation loss of grating-assisted directional coupler," IEEE J. Quantum Electron., vol. 26, pp. 675-684, Apr. 1990.

[18] D. Marcuse, "Directional couplers made of nonidentical asymmetric slabs. Part II: Grating-assisted couplers," J. Lightwave Technol, vol. LT-5, pp. 268-273, Feb. 1987.

[19] I. Kay, "The inverse scattering problem when the reflection coefficient is a rational function," Commun. Appl. Math, vol. 13,pp. 371393. 1960.

[20] K. R. Pechenick and J. M. Cohen, "Inverse scattering-exact solution of the Gel'fand-Levitan equation," J. Math. Phys., vol. 22, pp. 15131516, July 1981

[21] T. Tamir, Ed., Guided-Wave Optoelectronics. New York: SpringerVerlag, 1988 , ch. 2

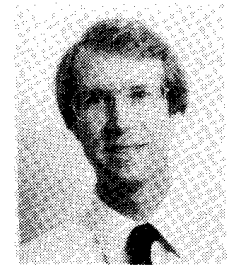

Kim A. Winick (S'77-M 80 ) was born in New York City on July 27,1954 . He received the B.S. degree in electrical engineering from the Pennsy! vania State University in 1976, and the M.S. and $\mathrm{Ph}$.D. degree in electrical engineering from the University of Michigan. Ann Arbor, in 1977 and 1981 , respectively. While at the University of Michigan, he held a National Science Foundation Graduate Fellowship.

From 1981 until 1988, he was a technical staft member at the Massachusetts Institute of Technology Lincoln Laboratory working on millimeter wave and coherent optical communications systems. Since the Fall of 1988, he has been an Assistant Professor in the Department of Electrical Engineering and Computer Science at the University of Michigan in Ann Arbor. His major research interests are in the areas of optical communications systems and integrated optics.

Dr. Winick is a member of the Optical Society of America. 